\title{
Molecular Structure of Magnesium Dibromide: An Electron Diffraction and Quantum Chemical Study
}

\section{Balázs Réffy, Mária Kolonits and Magdolna Hargittai ${ }^{1}$}

Structural Chemistry Research Group of the Hungarian Academy of Sciences, P.O.Box 32, H-1518

\author{
Budapest, Hungary
}

\section{Supporting Information}

Molecular intensities for two camera ranges of magnesium dibromide

Camera range $=19 \mathrm{~cm}$

\begin{tabular}{|c|c|c|c|c|c|c|c|}
\hline \multirow[t]{2}{*}{$\mathrm{s}_{\min }=9.00 \AA^{-1}$} & $S_{\max }=30.00 \AA^{-1}$ & \multicolumn{2}{|c|}{ step size $=0.25 \AA^{-}$} & & & & \\
\hline & & & & 147.4 & 23.9 & -75.3 & -103.9 \\
\hline-112.1 & -121.6 & -98.5 & -30.1 & 47.5 & 160.7 & 149.2 & 103.3 \\
\hline 21.9 & -42.7 & -64.6 & -85.7 & -93.4 & -81.0 & -33.3 & 34.2 \\
\hline 97.0 & 84.7 & 59.8 & 18.3 & -15.0 & -48.7 & -60.9 & -54.1 \\
\hline-52.1 & -11.0 & 5.0 & 43.7 & 58.1 & 57.7 & 25.3 & -6.7 \\
\hline-36.3 & -46.4 & -57.2 & -52.4 & 1.7 & 31.7 & 44.6 & 43.1 \\
\hline $4 \odot .1$ & 13.7 & -8.7 & -1.1 & -48.5 & -26.3 & -12.4 & -17.1 \\
\hline-4.9 & 41.4 & 23.0 & 3.5 & 9.2 & -19.8 & -5.7 & -7.1 \\
\hline-3.2 & -29.2 & .6 & -6.6 & 45.0 & 9.7 & 26.0 & -14.9 \\
\hline .0 & -26.2 & -1.0 & -19.6 & -11.7 & 7.1 & -17.4 & 21.8 \\
\hline 15.7 & 19.4 & 3.3 & -7.0 & -13.7 & -21.9 & 4.0 & 3.7 \\
\hline-1.3 & & & & & & & \\
\hline
\end{tabular}

Camera range $=50 \mathrm{~cm}$

$\mathrm{S}_{\min }=2.50 \AA^{-1} \mathrm{~S}_{\max }=14.00 \AA^{-1} \quad$ step size $=0.125 \AA^{-1}$

$\begin{array}{rrrrrrrr} & & & & -252.7 & -116.7 & 38.5 & 173.7 \\ 290.7 & 376.9 & 383.4 & 337.9 & 253.1 & 150.1 & 39.7 & -43.0 \\ -86.4 & -103.2 & -101.0 & -107.0 & -117.4 & -160.4 & -200.2 & -239.7 \\ -255.0 & -234.1 & -169.1 & -60.1 & 61.6 & 191.0 & 294.3 & 342.3 \\ 335.8 & 276.9 & 184.5 & 89.8 & 9.6 & -59.8 & -91.8 & -111.5 \\ -114.3 & -103.7 & -115.1 & -132.3 & -160.6 & -185.2 & -186.0 & -160.8\end{array}$

\footnotetext{
${ }^{1}$ To whom correspondence should be addressed. Email: hargitta@ludens.elte.hu
} 


$\begin{array}{rrrrrrrr}-100.7 & -21.7 & 73.6 & 160.0 & 211.1 & 249.7 & 246.2 & 207.8 \\ 154.0 & 74.4 & 12.3 & -39.6 & -79.6 & -89.2 & -102.9 & -101.3 \\ -111.9 & -120.4 & -126.4 & -121.5 & -115.0 & -86.8 & -51.8 & 10.9 \\ 59.2 & 101.8 & 149.1 & 166.0 & 163.6 & 140.9 & 96.7 & 56.1 \\ 13.5 & -11.7 & -45.7 & -60.4 & -70.2 & -68.2 & -84.6 & -77.7 \\ -93.6 & -86.7 & -85.0 & -56.5 & -28.0 & 0.4 & 43.8 & 75.4 \\ 105.3 & & & & & & & \end{array}$

Vibrational amplitudes [Á] of $\mathrm{MgBr}_{2}$ (linear) and $\mathrm{Mg}_{2} \mathrm{Br}_{4}\left(D_{2 h}\right.$ symmetry) calculated from the computed frequencies at the MP2/Mg: QZ, Br: QZ-PP level (see the paper for details)

\section{$\mathrm{MgBr}_{2}$}

$\begin{array}{ll}l(\mathrm{Mg}-\mathrm{Br}) & 0.091 \\ l(\mathrm{Br} \cdots \mathrm{Br}) & 0.125 \\ & \mathbf{M g}_{2} \mathrm{Br}_{4}\end{array}$

$l\left(\mathrm{Mg}-\mathrm{Br}_{\mathrm{t}}\right)$

$l\left(\mathrm{Mg}-\mathrm{Br}_{\mathrm{b}}\right)$

$l(\mathrm{Mg} \cdots \mathrm{Mg})$

$l\left(\mathrm{Br}_{\mathrm{b}} \cdots \mathrm{Br}_{\mathrm{b}}\right) \quad 0.174$

$l\left(\mathrm{Mg} \cdots \mathrm{Br}_{\mathrm{t}}\right) \quad 0.214$

$l\left(\mathrm{Br}_{\mathrm{b}} \cdots \mathrm{Br}_{\mathrm{t}}\right) \quad 0.306$

$\begin{array}{ll} & \left(\mathrm{Br}_{\mathrm{t}} \cdots \mathrm{Br}_{\mathrm{t}}\right) \quad 0.231\end{array}$ 